\title{
ASPECTOS MORFOLÓGICOS DE CILIADOS DE RÚMEN (ENTODINIOMORPHIDA, OPHRYOSCOLECIDAE) REVELADOS PELAS TÉCNICAS DO PROTARGOL E DA REAÇÃO DE FEULGEN
}

\author{
Marta D’Agosto 1 \\ Milden Rodrigues de Santa-Rosa ${ }^{2}$
}

\begin{abstract}
MORPHOLOGICAL ASPECTS OF RIIMIN CILIATE (ENTODINIOMORPHIDA, OPHRYOSCOLI(II)AE) DFMONSTRED BY PROTARgOL ANI) FELLGEN'S REATION TlCHNIQt Es. The results of the protargol's technique and Feulgen's reactions on siliates of the genus Entodinium Stein. 1859. Diplodinium Schuberg. 1888. Eodinium Kofoid \& MacLennan. 1932. Eudiplodinism Dogiel, 1927 and Ostracodinium Dogiel. 1927 are presented. The ciliates were ohtained from samples of hovine's ruminal content.

KEY WORDS. Entodiniomorphida. Ophryoseolecidae, rumen ciliate, silver impregnation. Feulgen's reaction
\end{abstract}

Desde a primeira observação por GRUBY \& DELAFOnd (1843), espécies de ciliados de rúmen vêm sendo assinaladas e descritas nas diversas regiões de ocorrêncra de seus hospedeiros, ruminantes e pseudo-ruminantes domesticados ou silvestres. Quanto às técnicas de evidenciação de stas estruturas são utilizadas montagens provisórias com ciliados não corados (V AN HoVEN 1983), em glicerina (KOFOID \& Maclennan 1930; Wertheim 1935; Das-Gupta 1935: Clarke 1964) ou corados. Para evidenciar o macronúcleo é comum o emprego do verde de metila (CLARKE 1964; DEhORITy 1974: IMAI 1988), verde de metilapironina-acéto-orceina-clorídrico ou reação ao Azur A. Gürr (CHARDEZ 1983) ou azul de metileno acidificado (DEHORITY 1974), e para evidenciação das placas esqueléticas solução de Lugol ou outra contendo iodo (KOFOID \& CHRISTENSON 1934; Das-Gupta 1935: Zielyk 1961; Clarke 1964: DehORITY 1974; VAN HOVEN 1975, 1983: CHARDEZ 1983).

Quanto às preparações permanentes, foram referidas a coloração pela hematoxilina de Heidenhain, Mallory, Delafield ou de Mayer, e ainda hematoxilina e eOSina (KOFOID \& MACLENNAN 1930, 1932; WERTHEIM 1935; DAS-GuPTA 1935; ZIELYK 1961: VAN HOVEN 1975, 1983; OGIMOTO \& IMAI 1981; IMAI 1988). Preparações com verde de metila desidratadas em álcool e xilol e montadas em Euparol foram feitas por CLARKE (1964), e com carmin borácico por ZIELYK (1961).

1) Departamento de Zoologia. Universidade Federal de Juis de Fora. 36036-330 Juis de Fora. Minas Gerais. Brasil.

2) Instituto de Microhiologia. Universidade Federal do Rio de Janeito. 21944-970 Rio de Janeiro. Rio de janciro. Brasil. 
OGIMOTO \& IMAI (1981) citaram como possíveis técnicas para preparações pernanentes de ciliados de rúmen, além da hematoxilina férrica de Heidenhaim, a impregnação pela prata, segundo Bodian, mencionando ainda a técnica de ChatTON-LWOFF (1930) simplificada, de acordo com Frankel \& HeCKMANN (1968).

ZIELYK (1961), além das técnicas já citadas, fez preparações utilizando nigrosina, $10 \%$ de azul opala, e técnicas de impregnação pela prata de KLEIN (1958) e ChATTON-LwOFF (1930) para evidenciar estriações longitudinais de organismos do gênero Entodinium Stein, 1859. O autor assinalou não haver obtido nenhum resultado positivo na coloração ou impregnação da infraciliatura.

O presente trabalho objetivou investigar o resultado da utilização de duas técnicas de preparações permanentes, o protargol e a reação de Feulgen, no estudo de ciliados de rúmen de bovinos por se tratar de técnicas clássicas em ciliatologia, de ampla aplicação e que raramente são utilizadas para preservação de Entodiniomorfos.

\section{MATERIAL E MÉTODOS}

Estudos morfológicos de ciliados de rúmen de bovinos foram realizados em microscopia fotônica empregando-se a reação de Feulgen (FEULGEN 1926) e técnica de Bodian (protargol) segundo a variante de TUFFRAU $(1964,1967)$. O princípio desta última consiste na utilização de sais de prata, proteinato de prata, como agente impregnante e posterior revelação em hidroquinona. Já na reação de Feulgen, após uma hidrólise ácida, os componentes nucleares coram-se de vermelho intenso por reação com a fuccina do preparado de Schiff.

A triagem dos ciliados foi feita em saleira, com o auxílio de micropipetas, a partir de amostras de conteúdo ruminal fixadas e conservadas em formalina a $18,5 \%(\mathrm{v} / \mathrm{v})$, sob microscópio estereoscópico com aumento entre 20 e 40x. As amostras foram filtradas em gaze para eliminação dos fragmentos vegetais maiores e lavadas sucessivamente com água destilada para eliminação dos resíduos de conteúdo ruminal e para concentração dos ciliados.

Para a técnica do protargol, a cada gota de água contendo ciliados era adicionada quantidade cinco vezes maior de Bouin Hollande como pós-fixador, o que faciliatava a visualização e triagem dos ciliados. $\mathrm{O}$ material foi lavado em água destilada até a eliminacão dos resíduos do Bouin e de vestígios de conteúdo ruminal. A seguir, separavam-se os ciliados, agrupando-os segundo suas dimensões (e.g., pequenos entodiniomorfos, grandes entodiniomorfos) em diversas saleiras, para clarificacão em água de Javel, com concentração e tempo variáveis de acordo com grupo de ciliados.

O tempo de impregnação no proteínato de prata, a $1 \%$, variou de 24 horas, para pequenos entodiniomorfos, a duas e três horas, para os grandes entodiniomorfos. As impregnações foram realizadas a temperatura ambiente.

Para a reação de Feulgen, os ciliados lavados, triados e concentrados por micropipetagem foram aderidos às lâminas com albumina glicerinada, preparada segundo FOISSNER (1991), antes de se proceder à hidrólise em ácido clorídrico a 
$1 \mathrm{~N}\left(14\right.$ a $\left.60^{\circ} \mathrm{C}\right)$ e submersão no reativo de Shiff por três horas.

As demais etapas, não mencionadas, seguiram o preconizado pela técnica correspondente até a montagem em bálsamo do Canadá.

As micrografias foram feitas em microscópio ZEISS-Germany com aumento de $1000 x$.

\section{RESULTADOS E DISCUSSÃO}

Apesar de rotineiramente utilizadas em ciliatologia, não é comum o emprego do protargol ou da reação de Feulgen para evidenciação de estruturas de ciliados de rúmen ou para sua preservação em coleções de referência.

O registro dos resultados obtidos com o protargol e a reação de Feulgen em ciliados ruminais dos gêneros Entodinium Ste in, 1859, Diplodinium Schuberg, 1888, Eodinium Kofoid \& MacLennan, 1932, Eudiplodinium Dogiel, 1927 e Ostracodinium Dogiel, 1927 pode ser observado nas figuras 1-5.

Com o protargol, os caracteres morfológicos principais para a identificação específica destes ciliados foram evidenciados como, por exemplo, a estrutura externa e suas particularidades como estrias e fendas na película (Fig. 1A, B), zonas de sincília (Figs 1-5), macro e micronúcleonúcleo (Fig. 1-5), vacúolo pulsátil (Figs 2B, 3A). Outros caracteres como o opérculo - quando presente -, citofaringe, citoprocto e fagossomo também podem ser observados (Figs 1-5). Registraram-se ainda, a impregnação do macronúcleo (Figs 1D, 3B) e do micronúcleo (Fig. 1D) em divisão e de novas zonas ciliares na formação do opisto (Fig. $3 \mathrm{~B}, 4 \mathrm{D}, 5 \mathrm{~A})$.

Destacam-se as melhores impregnações nos organismos de menores dimensões e em geral sem placas esqueléticas, excetuando-se os do gênero Eudiplodinium que apresentam pequena placa esquelética e hons resultados de impregnação. Todavia, para os ciliados cuja identificacão genérica é feita pelo número e forma das placas esqueléticas, em geral grandes entodiniomorfos, a impregnacão argêntica é inadequada por não evidenciar completamente estas estruturas, apenas vislumbrando-se sua presença em algunas preparaçoes (Fig. 5B). As melhores evidenciações das placas esqueléticas são obtidas com o emprego de soluções a base de iodo (CHARdez 1983; VAN Hoven 1975, 1983), apesar de serem preparações provisórias.

Para os entodiniomorfos de maiores dimensões como os dos gêneros Ostracodinium, Polyplastron Dogiel, 1927 e outros, o emprego do protargol é restrito pela excessiva impregnação destes organismos, observada mesmo com a redução do tempo de imersão no proteinato de prata. Em algumas preparações, entretanto, nota-se a nítida impregnação do macronúcleo e das zonas de sincília, protraídas ou não (Fig. 5A, C), como as observadas em Ostracodinium sp. Com os organismos de outros gêneros deste grupo não foram obtidos bons resultados.

Dentre as poucas referências à esta técnica para entodiniomorfos. OGIMOTO \& IMAI (1981) citam uma variante do protargol como um dos métodos para montagem permanentes de ciliados. Os autores não apresentaram, contudo, 

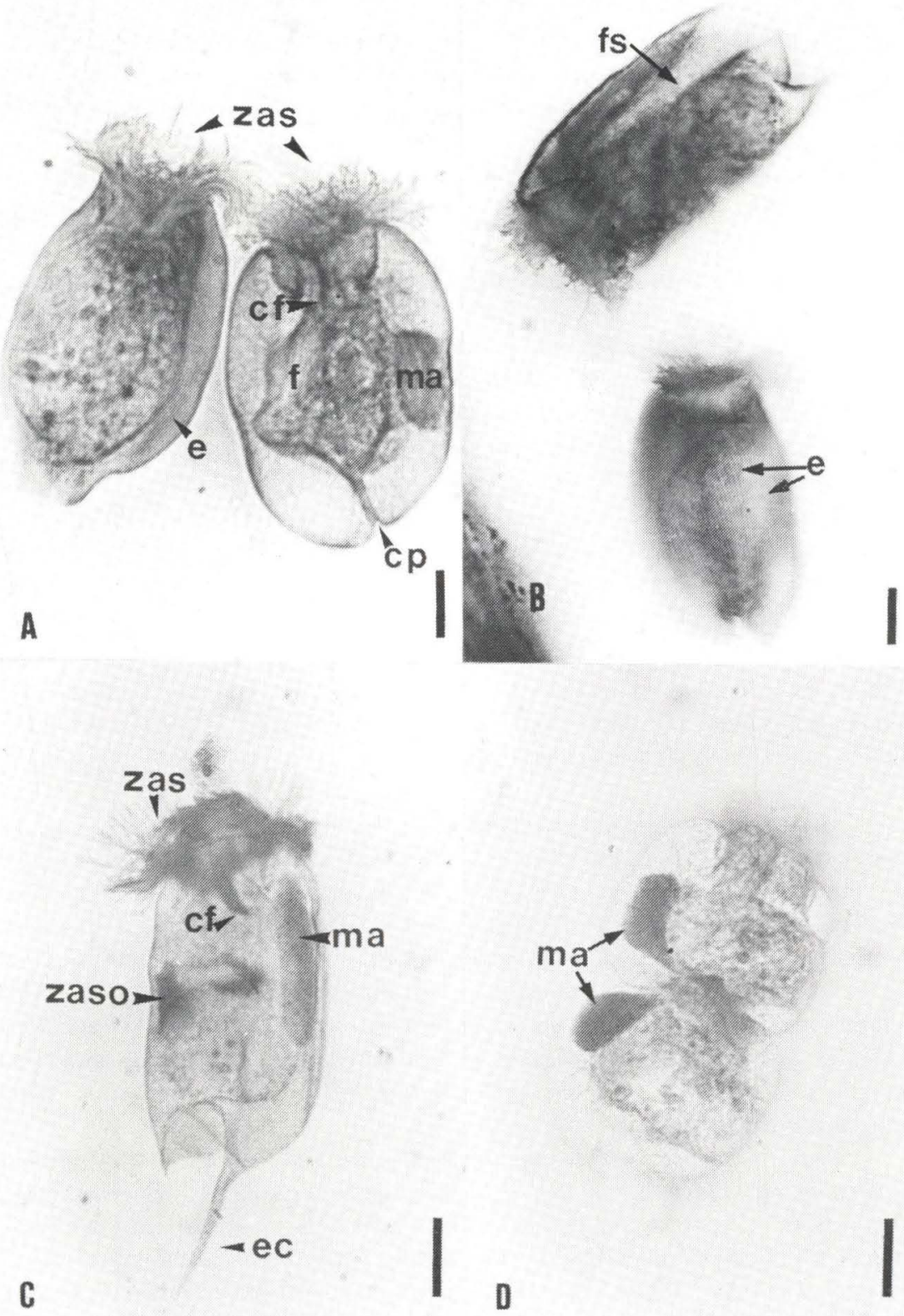

Fig. 1. Micrografias de Entodiniım spp. en Protargol (A, B, C) e reação de Feulgen (I)). Zona adoral de sincília (zas), Zona adoral de sincília do opisto em formação (zaso), macronúcleo (ma) em divisão na letra D, citofaringe (cf), fagossoma (f), citoprocto (cp), estrias (e), fenda superior (fs), espinho caudal (ec). Barra $=10 \mu \mathrm{m}$. 

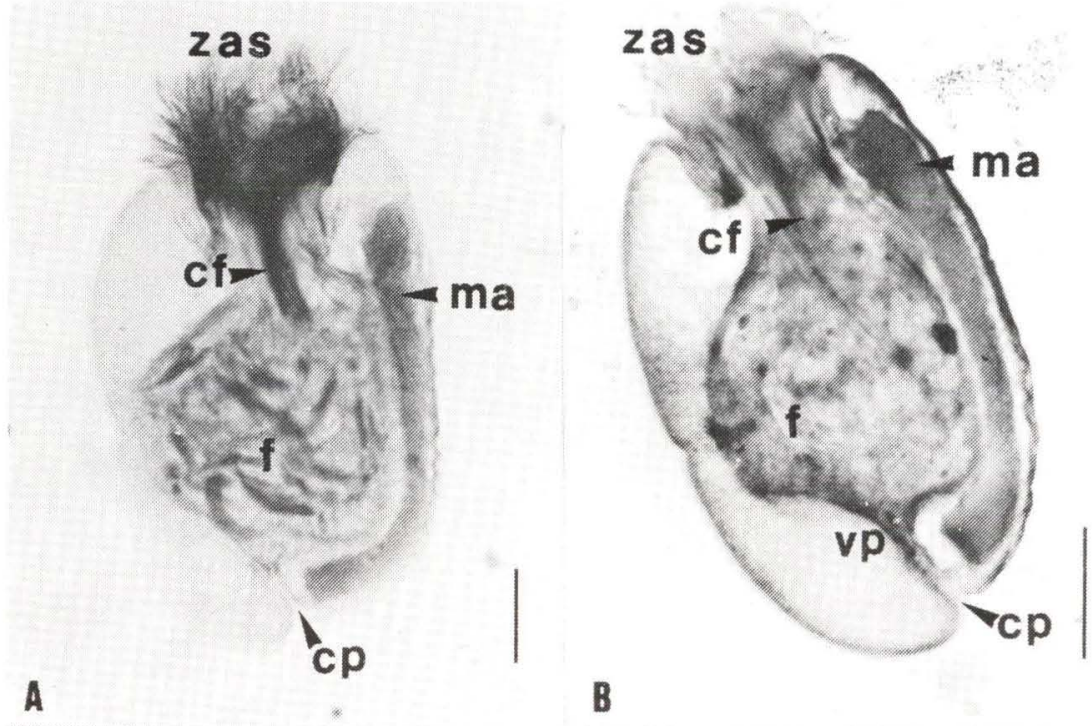

B

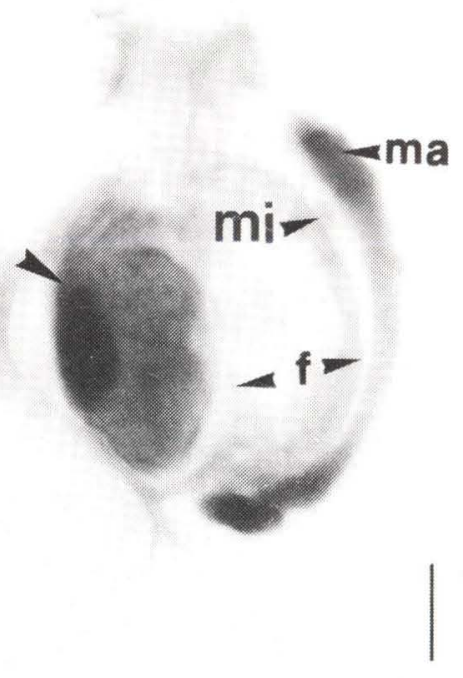

C

D

Fig. 2. Mierografias de Entodinium longinucleaum, em Protargol (A, B) e reação de Feulgen (C. D). Zona adoral de sincília (zas), macronúcleo (ma), micronúcleo (mi), fagossoma (f), citoprocto (cp), vacúolo pulsátil (vp) : (C) predação de um congênere (seta) : (D) restos de material nuclear de um congênere parcialmente digerido (setas). Barra $=10 \mu \mathrm{m}$. 

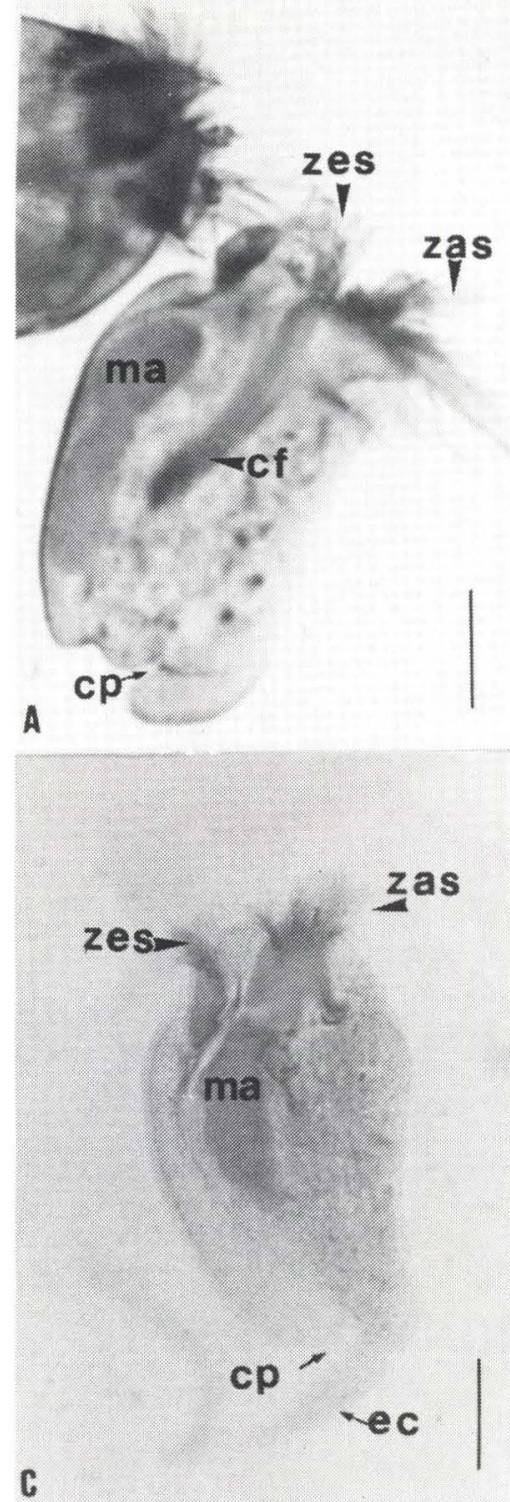

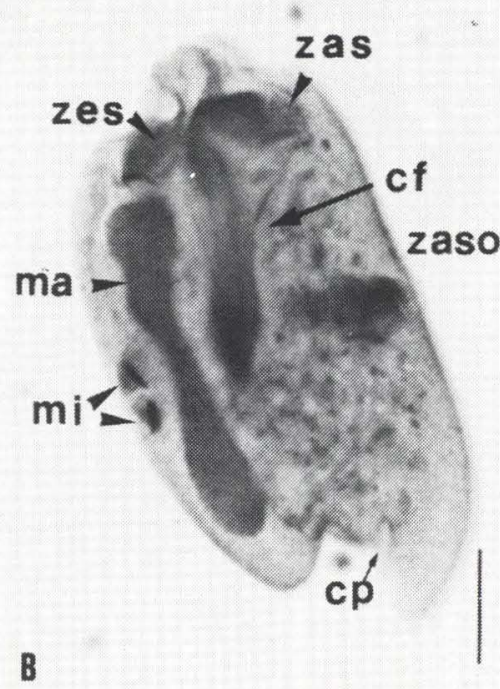

B

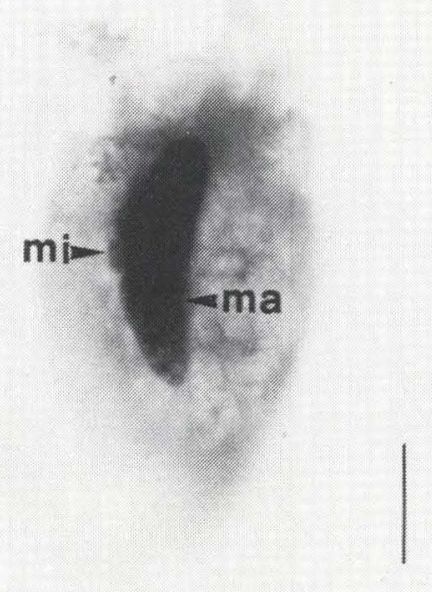

Fig. 3. Micrografias de Eodinium sp. em Protargol (A, B) e de Eudiplodinium rostratum em protargol (C) e reação de Feulgen (D). Zona adoral de sincília (zas), zona esquerda de sincília (zes), zona adoral de sincília do opisto em formação (zaso), zona esquerda de sincília do opisto em formação (zeso), macronúcleo (ma), micronúcleo (mi), citofaringe (cf), citoprocto (cp), vacúolo pulsátil (vp), opérculo (o). Barra $=10 \mu \mathrm{m}$. 


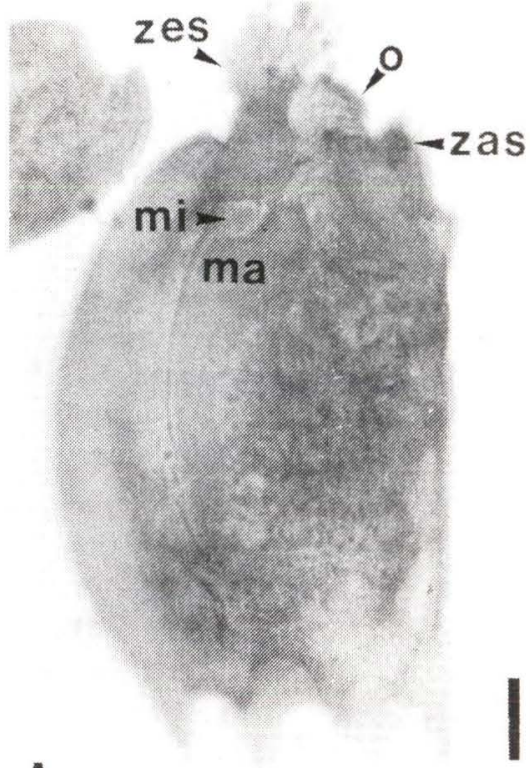

A

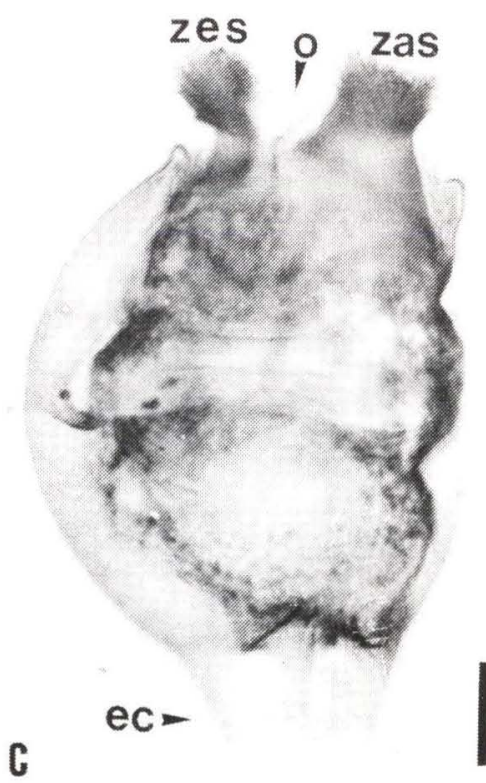

B
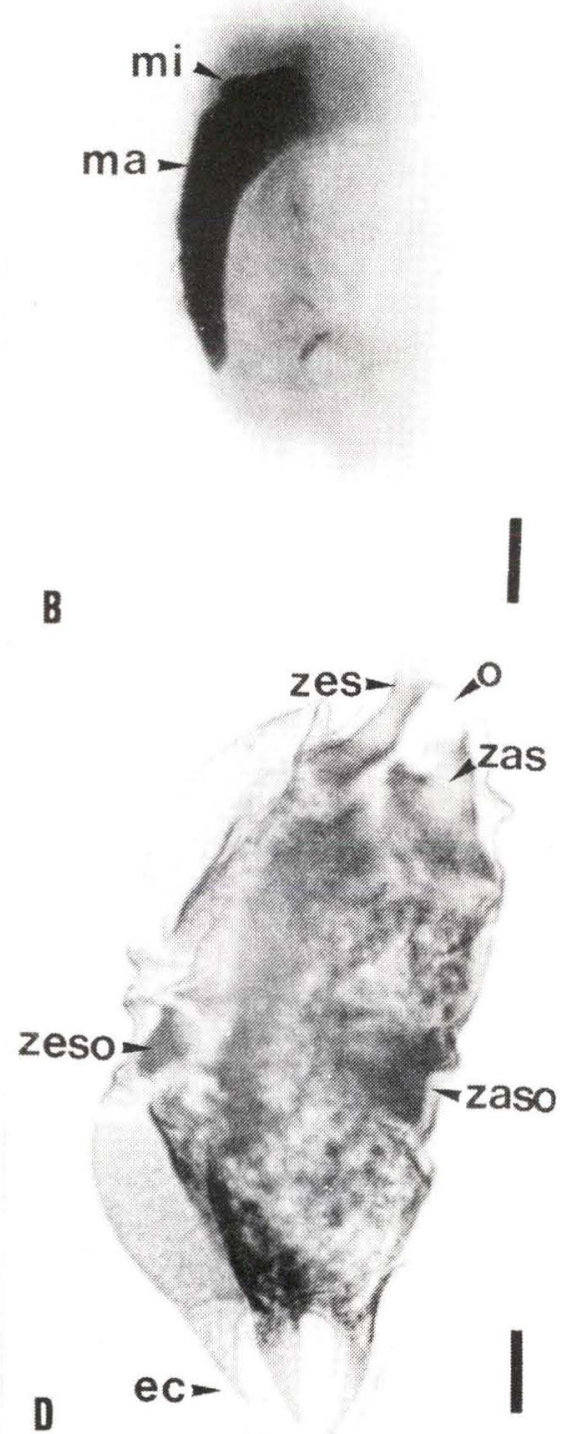

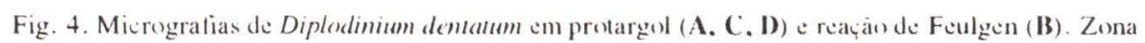
adoral de sincília (zas), zona esquerda de sincilia (zes), \%ona adoral de sincilia do opisto em formação (zaso), macronúcleo (ma). mieronúcleo (mi), espinhos caudais (ec), op̣érculo (o). Barra $=10 \mu \mathrm{m}$. 


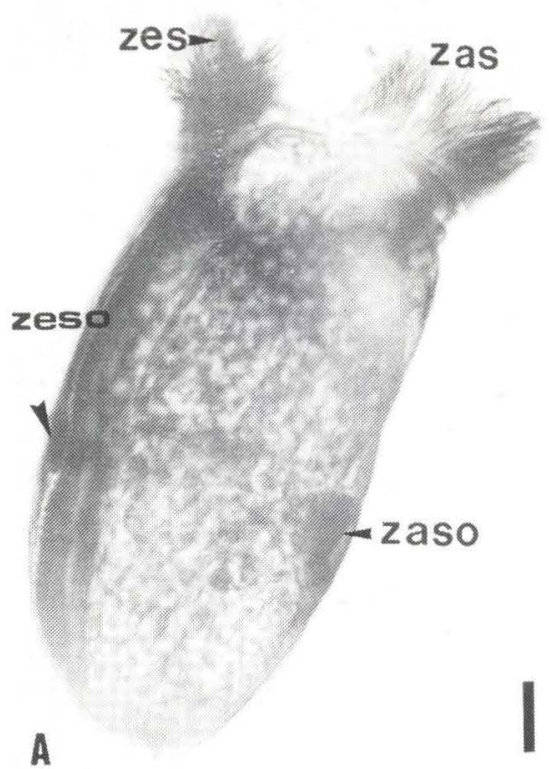

B

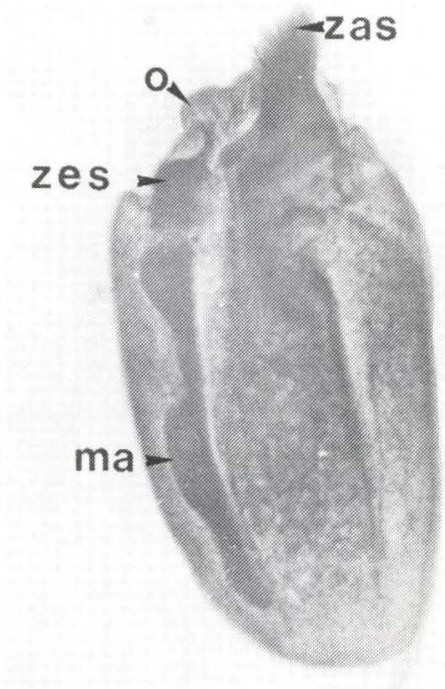

C
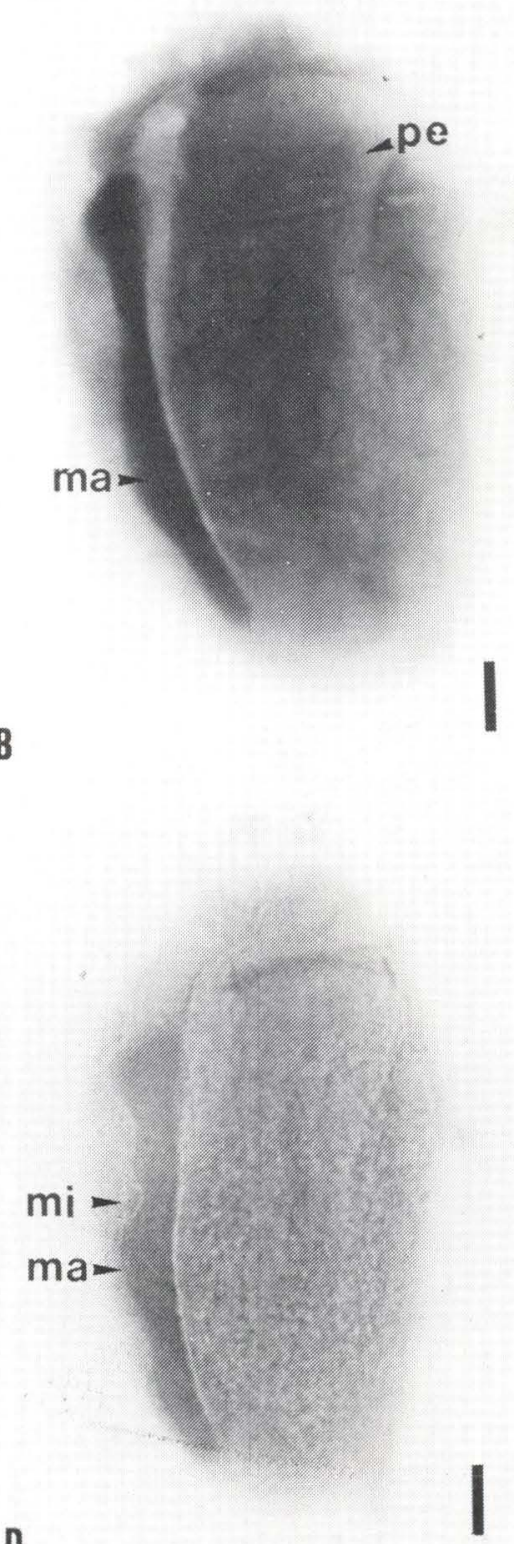

D

Fig. 5. Micrografias de Ostracodinium sp. em protargol (A, C) e reaçào de Feulgen (B, D). Zona adoral de sincília (zas), zona esquerda de sincília (zes) protraída em A e retraída em $\mathbf{C}$, zona adoral de sincília do opisto em formação (zaso), zona esquerda de sincília do opisto em formação (zeso), macronúcleo (ma), micronúcleo (mi), opérculo (o), vestígio de placa esquelética (pe). Barra=10ułum. 
resultados do seu emprego. Em estudo de organismos da família Ophryoscolecidae, especialmente de Entodinium duhardi Buisson, 1923 de cervídeo, ZIELYK (1961) utilizou técnicas de impregnação pela prata de KLEIN (1958) e de CHATTONLWofF (1930) assinalando não ter ohservado a impregnação da infraciliaturà. O autor concluiu que material fixado com formalina é inadeq̣uado a esta impregnação. A impregnação da infraciliatura de entodiniomorfos com carbonato de prata com piridina foi obtida por NoIROT-TIMOTHÉE (1960) e por FERNANDEZ-GALIANO) (1958), sem o emprego de piridina, em método que chamou de impregnação rápida. Este autor realizou estudo sobre Polyplastron multivesiculatum (Dogiel \& Fedorowa, 1925), ressaltando ter obtido hons resultados. Nos resultados obtidos no presente trabalho. em concordância com o observado por ZIELYK (1961). não houve a impregnaçào da infraciliatura.

Nas preparaçōes em que se utilizou a reação de Feulgen (Fiğs ID; 2C, D; 3D; 4B; 5B, D) nota-se que o objetivo da técnica, que é a evidenciação das estruturas nucleares, toi atingido sendo possível a observação do macro e do micronúcleo. Até mesmo nos grandes entodiniomorfos os resultados foram bastante relevantes como os demostrado em Ostracodinium sp. (Fig. 5B, D).

Credita-se à conservação prolongada do material em formalina a dificuldade de se obter a completa hidrólise do citoplasma, observando-se, em muitos organismos, uma coloração discreta do mesmo, o yue não é comum nesta técnica. Com o emprego da reação de Feulgen destacou-se ainda o háhito de predação de outros ciliados, aqui registrado para Entodinium longinuclestum Dogiel, $1925 \mathrm{com}$ outro organismo do mesmo gênero no interior de um fagossomo (Fig. 2C) e resto de material nuclear de ciliado já digerido (Fig. 2D). Graças à especificidade desta reação, pôde-se identificar como nucleares as estruturas ohservadas nestes fagossomos.

\section{CONCLUSÕES}

Concluiu-se que, apesar do long̨o tempo decorrido entre a ohtenção das amostras e o emprego das técnicas de impregnação e de coloração, é possível a sua utilização. Ressalta-se ainda, a importância de se preparar lâminas permanentes que sirvam de material de referência para o estudo destes ciliados, sobretudo quando novas espécies são descritas, a despeito da possihilidade de se observar algumas de suas estruturas em preparações näo coradas ou temporárias.

As duas técnicas empregadas evidenciaram particularmente hem as seguintes estruturas: núcleos (macro e micronúcleo), zonas de sincilia. citofaringe, citoprocto e fagossoma.

\section{REFERÊNCIAS BIBLIOGRÁFICAS}

Chardez, D. 1983. Sur la variabilité de deux espèces d'Ophryoscolecidae (Ciliophora, Entodiniomorphida). Bull. Rech. Agron. Gembloux 18: 257264.

Chatton, E. \& A. LWoff. 1930. Impregnation, par diffusion argentique de 
l'infraciliatura des ciliés marins et d'eau douce, après fixation cytologique et sans dessication. C.R. Soc. Biol. 104: 834-836.

Clarke, R.T.J. 1964. Ciliates of the rumen of domestic cattle (Bos taurus L.). N.Z.J. Agric. Res. 7: 248-257.

Das-Gupta, M. 1935. Preliminary observations on the protozoan fauna of the rumen of the indian goat, Capra hircus Linn. Arch. Protist. 85: 153-172.

DEHORITY, B.A. 1974. Rumen ciliate fauna of alaskan moose (Alces americana), musk-ox (Ovibos moschattus) and dall mountain sheep (Ovis dalli). J. Protozool. 21: 26-32.

Fernandez-Galiano, D. 1958. La infraciliación en Polyplastron multivesiculatum y su génesis durante la división del ciliado. Bol. R. Soc. Esp. Hist. Nat. (Secc. Biol.) 56: 89-108.

FRANKEL, J. \& K. HECKMANN. 1968. A simplified Chatton-Lwoff silver impregnation procedure for use in experimental studies with ciliates. Trans. Amer. Microsc. Soc. 87: 317-321.

FEulgen, R. 1926. Die nuclealfarburg abderharden's. 1926. Haudb. biol. Arbests, meth Abt, 5: 1055.

FOISSNER, W. 1991. Basic ligth and scanning electron microscopic methods for taxonomic studies of ciliate protozoa. Europ. J. Protistol. 27: 313-330.

Gruby, D. \& O. Delafond. 1843. Recherches sur des animalcules se développant en grand nombre dans l'estomac et dans les intestins, pendant la digestion des animaux herbivores et carnivores. Compt. Rend. Acad. Sci. 17: 1304-1308.

IMAI, S. 1988. Ciliate protozoa in the rumen of kenyan zebu cattle, Bos taurus indicus, with the description of four new species. J. Protozool. 35: 130-136.

KLEIN, B. M. 1958. The "dry" silver method and its proper use. J. Protozool. 5: 99-103.

Kofoid, C. A. \& J.F. Christenson. 1934. Ciliates from Bos gaurus. Univ. Calif. Publ. Zool. 39: 341-391.

Kofoid, C.A. \& R.F. Maclennan. 1930. Ciliates from Bos indicus Linn. I. The genus Entodinium Stein. Univ. Calif. Publ. Zool. 33: 471-544.

1932. Ciliates from Bos indicus Linn. II. A revision of Diplodinium Schuberg. Univ. Calif. Publ. Zool. 37: 53-152.

NorRot-Timothée, C. 1960. Étude d'une famille de ciliés: les Ophryoscolecidae. Strutures et ultrastructures. Ann. Sci. Nat. Zool. 2: 527-718.

Ogimoto, K. \& S. Imai. 1981. Atlas of rumen biology. Tokio, Scientific Societies Press, VIII +231p.

Tuffrau, M. 1964. Quelques variantes techniques de l'impregnation des ciliés par le Protéinate d'argent. Arch. Zool. Exp. 104: 186-190.

— 1967. Perfectionnement et pratique de la technique d'impregnation au Protargol des infusoires ciliés. Protistologica 3: 91-98.

Van Hoven, W. 1975. Rumen ciliates of the tsessebe (Damaliscus lunatus lunatus) in South Africa. J. Protozool. 22: 457-462.

1983. Rumen ciliates with description of two new species from three 
african reedbuck species. J. Protozool. 30: 688-691.

Wertheim, P. 1935. A new ciliate, Entodinium bovis n. sp., from the stomach of Bos taurus L. with the revision of Entodinium exiguum, E. nanellum, $E$. simplex, E. dubardi dubardi and E. parvum. Parasitology 27: 226-230.

ZIELYK, M.W. 1961. Ophryoscolecid fauna from the stomach of the white-tailed deer (Odocoileus virginianus borealis), and observations on the division of Entodinium dubardi Buisson, 1923 (Ciliata Entodiniomorpha). J. Protozool. 8:33-41.

Recebido em 15.III.1993; aceito em 22.IV.1994. 